\title{
Error analysis of nuclear forces and effective interactions
}

\author{
R. Navarro Pérez, J.E. Amaro and E. Ruiz Arriola \\ Departamento de Física Atómica, Molecular y Nuclear \\ and Instituto Carlos I de Física Teórica y Computacional \\ Universidad de Granada, E-18071 Granada, Spain. \\ E-mail: rnavarrop@ugr.es, amaro@ugr.es, earriola@ugr.es
}

\begin{abstract}
The Nucleon-Nucleon interaction is the starting point for ab initio Nuclear Structure and Nuclear reactions calculations. Those are effectively carried out via effective interactions fitting scattering data up to a maximal center of mass momentum. However, NN interactions are subjected to statistical and systematic uncertainties which are expected to propagate and have some impact on the predictive power and accuracy of theoretical calculations, regardless on the numerical accuracy of the method used to solve the many body problem. We stress the necessary conditions required for a correct and self-consistent statistical interpretation of the discrepancies between theory and experiment which enable a subsequent statistical error propagation and correlation analysis. We comprehensively discuss an stringent and recently proposed tail-sensitive normality test and provide a simple recipe to implement it. As an application, we analyze the deduced uncertainties and correlations of effective interactions in terms of Moshinsky-Skyrme parameters and effective field theory counterterms as derived from the bare NN potential containing One-Pion-Exchange and Chiral Two-Pion-Exchange interactions inferred from scattering data.

PACS numbers: 03.65.Nk,11.10.Gh,13.75.Cs,21.30.Fe,21.45.+v
\end{abstract}

Keywords: NN interaction, One Pion Exchange, Chiral Two Pion Exchange, Statistical Analysis, Effective Interactions

\section{Introduction}

Quantum Chromodynamics (QCD) is the underlying $S U\left(N_{c}\right)$ theory of $N_{c}$ coloured quarks and $N_{c}^{2}-1$ gluons $\left(N_{c}=3\right)$ which is expected to explain all known hadronic phenomena from the structure of the pion to the binding and interactions among atomic nuclei. For light quarks and disregarding electroweak interactions one expects this to be done in terms of just two scales: the pion weak decay constant $f_{\pi}$ and the pion mass $m_{\pi}$. The complexity of the problem for finite nuclei, neutron and nuclear matter has distorted the very usage of the term ab initio calculations [1]; instead of referring to the solution from QCD in terms of quarks, gluons and their interactions, it is meant the solution of the many-nucleon problem from the phenomenological knowledge of the two and/or three nucleon systems. 
This distinguishes phenomenological but realistic nucleon-nucleon interactions as the starting point for any $a b$ initio nuclear structure and nuclear reactions calculations, a long-standing framework whose parentage to QCD may be sought either by direct lattice QCD calculations 2 or indirect QCD NN-features such as chiral symmetry within an Effective Field Theory (EFT) approach [3, 4, 5, 6, 7, 8, 9, or large $N_{c}$ scaling of the NN potential [10, 11, 12, 13, 14. Any approach, even when assisted by these fundamental constraints, must obviously be validated by confronting $\mathrm{NN}$-forces to scattering data and deuteron properties before pursuing studies in heavier nuclei. This requires, in particular, the selection of a CM momentum range $p \leq \Lambda$ fixing a de Broglie resolution scale $\Delta r=\hbar / \Lambda$ and which can be suitably tuned to target the relevant effective coarse grained interaction [15] operating in a finite nucleus.

For finite number of fitting data with finite experimental accuracy, neither the theoretical phenomenological interaction nor the experimental data are free from inconsistencies and systematic bias. This issue becomes more acute when the number of data and model parameters is large. There is of course no infallible method to discard some subset of inconsistent data out of a finite number of experiments with finite precision, nor a subset of phenomenological interactions on the basis of a quantitative disagreement between theory and experiment. However, statistical methods allow to check within a prescribed probabilistic confidence level when both the most likely theory and a given set of experiments differ by just statistical fluctuations. This most often happens when the total set of data is reduced after some, presumably inconsistent, data are discarded and the model representing the data contains a sufficiently large number of independent parameters [16, 17, 18, 19, 20].

Data fitting by least squares minimization is a broadly used technique in phenomenological approaches in nuclear physics and more generally in all experimental sciences with satisfactory results. Much has been said on this topic and we refer to a recent and readable overview of the subject 21. In this paper we concentrate on the applicability conditions and assumptions underlying the procedure. While our remarks have general validity we will discuss the particular case of nuclear forces, and more specifically NN scattering below pion production threshold [22, 23, 24, 25]. Our presentation relies heavily on these works where further details may be found.

The goodness of a least squares fit is decided on the value of $\chi^{2} / \nu$ in probabilistic terms. Thus, one estimates the probability of discarding a theoretical model assuming it was a true one, a so-called type I error, see Sect.2.2 below. A key assumption which, if fulfilled, provides a sound mathematical basis for confidence level estimates and statistical error propagation is the normality of residuals measuring the discrepancy between the fitting model and the fitted data. For a finite number of data one can only give an answer to this question in probabilistic terms, and of course the conditions to answer in the affirmative are increasingly stringent with the number of fitted data. We want to stress that despite the elementary textbook character of this normality test [26], this part of the least-squares fitting is too often overlooked, perhaps because it can only be made a posteriori once the minimization has been finished and potentially

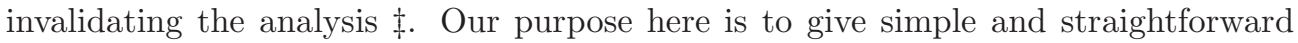
tools enabling such an analysis and hopefully to popularize it. More specifically, we quickly overview the normality testing and provide a convenient method which focuses on the selection process of mutually inconsistent data.

$\ddagger$ Many canned routines for fitting (for example MINUIT [27, POUNDERS [28]) of current use do not include this useful test at the moment of writing this contribution. 
As mentioned, phenomenological interactions are obtained by fitting scattering data up to a maximum CM momentum $\Lambda$, and thus there is an inherited $\Lambda$ dependence. We address here a remark in 21] "We are dealing almost everywhere with effective theories justified in terms of general arguments, but whose parameters are basically unknown and often cannot be deduced from ab-initio modeling". In fact, coarse graining the interaction down to the relevant scale allows to carry the identification of relevant parameters. This is the idea underlying the $V_{\text {lowk }}$ approach 29, 30, which universalizes the interaction and can be visualized from a Wilsonian renormalization point of view by means of the Block-Diagonal Similarity Renormalization group (BDSRG) equations [31. As it was shown in Ref. 32 for the NN problem and also in the case of diatomic systems [33, effective couplings can indeed be obtained just from scattering properties and for $\Lambda \lesssim 1 / a$, with $a$ the range of the interaction. The complementarity of this method with the full BD-SRG approach for $\Lambda \lesssim 200 \mathrm{MeV}$ has been checked 34. A different approach, directly fitting parameters up to a maximum energy yields very similar results for these $\Lambda$-values [35. Here we provide error estimates and correlations on the parameters of the effective interactions when fitting up to CM momentum $p \lesssim \Lambda=400 \mathrm{MeV}$.

One of the applications of the normality test discussed previously is that statistical error propagation including correlations may confidently be undertaken. In a series of papers we have addressed this issue concerning the NN interaction itself, nuclear matrix elements and possible implications for the accuracy of binding energies [36, 37, 38. An error analysis of the empirical mass formula was first discussed in Ref. [39. The Predictive power and theoretical uncertainties of mathematical modelling for nuclear physics has been studied in Ref. 40] within mean field theory approaches. Here we will focus on the expected statistical uncertainties of effective interactions in the $\mathrm{NN}$ sector.

\section{Statistical framework}

\subsection{Self-consistent least squares fit}

Given $N$ experimental observations $O_{i}^{\exp }$ with estimated errors $\Delta O_{i}^{\exp }$ the traditional figure of merit for a theory with $P$ parameters $\mathbf{p}=\left(p_{1}, \ldots, p_{P}\right)$, predicting theoretical observable values $\mathcal{O}_{i}(\mathbf{p})$, corresponds to minimizing the least squares sum

$$
\min _{\mathbf{p}} \chi^{2}(\mathbf{p})=\min _{\mathbf{p}} \sum_{i=1}^{N}\left(\frac{O_{i}^{\exp }-O_{i}(\mathbf{p})}{\Delta O_{i}^{\exp }}\right)^{2} \equiv \chi^{2}\left(\mathbf{p}_{0}\right) .
$$

Then the most likely theory parameters are $\mathbf{p}_{0}$, and the most likely theoretical prediction is $\mathcal{O}_{i}^{\text {th }} \equiv O_{i}\left(\mathbf{p}_{0}\right)$. The residuals at $\mathbf{p}_{0}$ are defined by

$$
R_{i}=\frac{O_{i}^{\exp }-O_{i}\left(\mathbf{p}_{0}\right)}{\Delta O_{i}^{\exp }}
$$

If $R_{i}$ obey a standardized normal distribution, i.e., the probability of obtaining residuals in the interval $[a, b]$ is

$$
P\left(a \leq R_{i} \leq b\right)=\int_{a}^{b} N(x) d x, \quad N(x)=\frac{e^{-x^{2} / 2}}{\sqrt{2 \pi}},
$$

then $\chi^{2}\left(\mathbf{p}_{\mathbf{0}}\right)$ follows a $\chi^{2}$-distribution with $\nu=N-P$ degrees of freedom. A confidence region in the parameter space around $\mathbf{p}_{0}$ may be defined by the set of values $\Delta \mathbf{p}$ such 
that $\chi^{2}\left(\mathbf{p}_{0}+\Delta \mathbf{p}\right)-\chi^{2}\left(\mathbf{p}_{0}\right)=\Delta \mathbf{p}^{T} \mathcal{E}^{-1} \Delta \mathbf{p} \leq 1$, where $\mathcal{E}_{i j}$ is the error matrix. The correlation $\mathcal{C}_{i j}$ matrix is defined as

$$
\mathcal{C}_{i j}=\frac{\mathcal{E}_{i j}}{\sqrt{\mathcal{E}_{i i} \mathcal{E}_{j j}}} .
$$

Thus, for any function of the parameters $F(\mathbf{p})$ one has that to $1 \sigma$ confidence level

$$
F(\mathbf{p})=F\left(\mathbf{p}_{0}\right) \pm \Delta F, \quad(\Delta F)^{2}=\left.\nabla_{\mathbf{p}} F^{T} \mathcal{E} \nabla_{\mathbf{p}} F\right|_{\mathbf{p}=\mathbf{p}_{0}} .
$$

Being $\chi^{2}$ a positive function the minimum always exists; the relevant issue concerns the requirement for a meaningful interpretation. As mentioned, the least squares method rests on the major assumption that discrepancies between theory and experiment are random normal variables. Thus, we have to decide whether any of the $R_{i}$ individually fulfills Eq. (3), a question which could only be answered by repeating the measurement. Instead, one decides for the ensemble $\left(R_{1}, \ldots, R_{N}\right)$ as a whole, a question which for a sample with finite size $N$ can only be answered in probabilistic terms and a posteriori, i.e. after the least squares fit has been obtained. In the case of scattering experiments we deal with a Poissonian statistics counting, which for moderately large number of counts becomes a gaussian distribution, so we do expect in the absence of systematic errors in the measurements and the theory the $R_{i}$ behaving as normalized gaussians. When this happens we have a self-consistent fit and we can write

$$
\mathcal{O}_{i}=\mathcal{O}_{i}^{\text {th }}+\xi_{i} \Delta \mathcal{O}_{i},
$$

where $\xi_{i}$ are standardized normal uncorrelated variables, and $\Delta \mathcal{O}_{i}$ are the experimental uncertainties. This formula has the virtue of simulating a synthetic set of individual and independent measurements. This has been recently exploited for a study of errors in fitting parameters of the $\mathrm{NN}$ force [41] and applied to estimate the statistical error in the theoretical binding energy of the triton [42].

\subsection{Normality tests}

For a set on $N$ empirical data $X_{i}$ the null hypothesis $H_{0}$ of a normality test is that the data follows a standard normal distribution i.e. $H_{0}: X_{i} \sim N(0,1)$. Correspondingly, the alternative hypothesis $H_{1}$ states that the empirical data follows a distribution $F_{1}$ different from $N(0,1)$. The test consists of quantitatively assessing if certain discrepancies between the empirical data distribution and $N(0,1)$ are large enough to confidently reject $H_{0}$; these discrepancies are quantified using a test statistic $T$. The decision to reject (or not) is made by comparing the observed test statistic $T_{\mathrm{obs}}$, which is calculated using the empirical data $X_{i}$, against the distribution of $T$ for random samples of $N(0,1)$ with size $N$. Each normality test has its own definition of $T$ and in some cases the distribution of $T$ under the null hypothesis is known analytically. When comparing $T_{\mathrm{obs}}$ to the distribution of $T$ a significance level $\alpha$ is arbitrarily chosen, this determines a critical value $T_{c}$ and $H_{0}$ is rejected if $T_{\text {obs }}$ is greater (or smaller, depending on the distribution of $T$ ) than $T_{c}$. Common choices for $\alpha$ are 0.01 and 0.05 . Another relevant quantity commonly quoted in normality tests is the $p$-value and corresponds to the smallest significance level at which $H_{0}$ would be rejected. A small $p$-value indicates clear deviations from normality, whereas a large $p$-value indicates that no statistically significant discrepancies where found.

As with any test, one of two type of errors can occur when a decision is made. One is giving a false negative, also known as type I error, which consists on rejecting $H_{0}$ when the data do follow the normal distribution; the other is a false positive, or 
type II error, which is made when $H_{0}$ is not rejected and the data follows a different distribution. The probability of a type I error is given by the value $\alpha$ and therefore can be arbitrarily fixed. With this in mind one would like $\alpha$ to be small enough to avoid a false negative; but not so small that almost any set of empirical data passes the test, rendering the test useless. The probability of a type II error (or false positive rate) is denoted by $\beta$ and is directly related to the power of the test, given by $1-\beta$. The statistical power is an intrinsic property of the test that is more appropriately analyzed a priori by applying the test to random samples drawn from non normal distributions (in this case one knows that the null hypothesis is false) and seeing how often $H_{0}$ is not rejected. The power of a test usually increases with the sample size $N$, and therefore a proper power analysis is needed to know how large should $N$ be to avoid giving a false positive.

\subsection{Finite sample statistical fluctuations of residuals}

If $X$ is a continuous random variable following a given probability distribution $\rho(x)$, the Cumulative Distribution Function (CDF) is defined as

$$
p(x) \equiv P(X<x)=\int_{-\infty}^{x} \rho(t) d t
$$

Clearly $p(-\infty)=0$ and $p(\infty)=1$. For example, the CDF of the standardized normal distribution is given by

$$
\Phi(x)=\int_{-\infty}^{x} N(t) d t=\frac{1}{2}\left[\operatorname{erf}\left(\frac{x}{\sqrt{2}}\right)+1\right] .
$$

If we generate $N$ independent data $x_{i}$ following a certain distribution $\rho(x)$ we define the empirical CDF (ECDF) as the fraction of data that is smaller then a certain value $x$ i.e.

$$
S_{N}(x)=\frac{1}{N} \sum_{i=1}^{N} \theta\left(x-x_{i}\right) .
$$

Of course the specific ECDF depends on the on the particular sample $\left(x_{1}, \ldots, x_{N}\right)$;

if we take $M$ different extractions $\left(x_{1}^{(i)}, \ldots, x_{N}^{(i)}\right), i=1, \ldots, M$, then $M$ different ECDF's $S_{N}^{(i)}(x)$ will be generated. Thus, for a function of these $N$ random variables $O\left(x_{1}, \ldots, x_{N}\right)$ we can define as usual the expected value as the arithmetic mean in the limit of $M \rightarrow \infty$

$$
\begin{aligned}
E[O] & \equiv \lim _{M \rightarrow \infty} \frac{1}{M} \sum_{i=1}^{M} O\left(x_{1}^{(i)}, \ldots, x_{N}^{(i)}\right) \\
& =\int_{-\infty}^{\infty} d x_{1} \rho\left(x_{1}\right) \ldots \int_{-\infty}^{\infty} d x_{N} \rho\left(x_{N}\right) O\left(x_{1}, \ldots, x_{N}\right) .
\end{aligned}
$$

One can thus compute the probability density of having a certain value $S$ of $S_{N}(x)$. Actually, since by definition we have $S_{N}(x)=n_{N}(x) / N$ with $n_{N}(x)$ an integer number $0 \leq n_{N}(x) \leq N$, we may instead compute the expected probability of finding a given value $n_{N}(x)=m$ as

$$
\begin{aligned}
P_{N, x}(m) & =E\left(\delta_{m, n_{N}(x)}\right)=E\left(\frac{1}{2 \pi} \int_{0}^{2 \pi} d \varphi e^{i \varphi\left(m-n_{N}(x)\right)}\right) \\
& =\frac{1}{2 \pi} \int_{0}^{2 \pi} d \varphi e^{i \varphi m}\left[p(x)+e^{-i \varphi}(1-p(x))\right]^{N},
\end{aligned}
$$


which after expanding the binomial and computing the integral becomes a binomial distribution

$$
P_{N, x}(m)=\left(\begin{array}{c}
N \\
m
\end{array}\right) p(x)^{m}[1-p(x)]^{N-m} .
$$

The expected value and the variance are thus

$$
E[m]=p(x) \quad(\Delta m)^{2}=\frac{1}{N} p(x)(1-p(x)) .
$$

Matters become simpler for large samples, $N \gg 1$, the binomial becomes a normal distribution

$$
P_{N, x}(m) \rightarrow \frac{1}{\sqrt{2 \pi} \Delta m(x)} \exp \left[-\frac{1}{2}\left(\frac{m / N-p(x)}{\Delta m(x)}\right)^{2}\right],
$$

so that we may write with $n \sigma$ confidence level

$$
S_{N}(x)=p(x) \pm \frac{n}{\sqrt{N}} \sqrt{p(x)(1-p(x))} .
$$

\subsection{Rotated Quantile-quantile plot}

The mapping

$$
z=p(x)=\int_{-\infty}^{x} \rho(t) d t
$$

transforms any distribution into the uniform one $U[0,1]$ since $d z=\rho(x) d x$ and $0<z<1$. For a set of $N$ uniformly distributed discrete points $z_{n}=n /(N+1)$ with $n=1, \ldots, N$ we define their corresponding $\rho$-theoretical values are defined as

$$
\frac{n}{N+1}=p\left(x_{n}^{\mathrm{th}}\right)=\int_{-\infty}^{x_{n}^{\mathrm{th}}} d t \rho(t)
$$

which fulfill $x_{1}^{\text {th }}<\ldots<x_{N}^{\text {th }}$ since the mapping is monotonous $d z / d x=\rho(x)>0$. The empirical quantile-quantile (QQ)-plot of the ranked set of points $x_{1}^{\exp }<\ldots<x_{N}^{\exp }$ is defined by plotting the points $\left(x_{n}^{\exp }, x_{n}^{\text {th }}\right)$ for $n=1, \ldots, N$. One expects that if the empirical points follow the distribution $\rho(x)$ then in the limit of $N \rightarrow \infty$ the QQplot should become a straight $\operatorname{line}_{\lim } \lim _{N \rightarrow \infty}\left(x_{n}^{\exp }-x_{n}^{\text {th }}\right)=0$. For finite $N$ there are, however, finite size fluctuations and therefore a departure from the straight line.

How large can be $\Delta x_{n}^{\exp }=x_{n}^{\exp }-x_{n}^{\text {th }}$ be before we suspect that $x_{n}^{\exp }$ do not follow the distribution $\rho(x)$ ?. For large $N$ we can use the normal distribution $N(x)$, Eq. (8), and fluctuations are more clearly displayed in terms of the rotated QQ plot. At the $1 \sigma$ confidence level

$$
\Delta x_{n}= \pm \sqrt{\frac{p\left(x_{n}\right)\left(1-p\left(x_{n}\right)\right)}{N}} \frac{1}{N\left(x_{n}\right)},
$$

where we have explicitly used that $x_{n}^{\exp }-x_{n}^{\text {th }}=\mathcal{O}(1 / \sqrt{N})$ to estimate the r.h.s. This is a quick method providing point-wise normality test bands when $N \gg 1$ in a rotated QQ-plot. In Ref. 25] we have applied a variety of traditional methods including the Pearson test, the Kolmogorov-Smirnov (KS) test, the moments method and more recent Tail sensitive (TS) test 43 , which we describe in the next subsection in a simplified way. We illustrate the situation for the rotated-QQ-plots in Fig. 1 (see discussion below) with the band suggested by Eq. (18) when we take as the empirical $x_{i}$ 's the residuals corresponding to the $\mathrm{NN}$ analysis. 


\subsection{Tail sensitive test}

The idea behind the tail sensitive (TS) test comes from the QQ-plot. The Monte Carlo scheme proposed in Ref. [43] was pursued in Ref. 25]. Here we provide a shortcut to avoid the large number of samples necessary for the MonteCarlo simulation. The recipe for checking normality, or any probability distribution, $\rho(x)$, for a empirical sample $x_{1}<\ldots<x_{N}$ of size $N$, consists of a few steps

(i) Decide what is the significance level $\alpha$ (typically 0.05 or 0.01 ), which is the probability of type I error, i.e. giving a false negative.

(ii) Determine the critical test statistic $T_{c}$, which for $N>50$ corresponds to

$$
T_{c}=\frac{a}{\sqrt{N}}+b
$$

where $a$ and $b$ depend on $\alpha$ and can be looked up in table A2 For $N<50$ one can look at table A1.

(iii) Transform the empirical data $x_{1}<\ldots<x_{N}$ to the new data $z_{1}<\ldots<z_{N}$ through the mapping

$$
z_{i}=\int_{-\infty}^{x_{i}} d t \rho(t)
$$

(iv) Compute the test statistic $T=2 \min _{i}\left\{\min \left[B_{i, N+1-i}\left(z_{i}\right), 1-B_{i, N+1-i}\left(z_{i}\right)\right]\right\}$, where the cumulative distribution function corresponds to the regularized incomplete Beta-function and is defined as

$$
B_{i, N+1-i}(z)=\sum_{j=i}^{N}\left(\begin{array}{c}
N \\
j
\end{array}\right) z^{j}(1-z)^{N-j}
$$

(v) Compare the observed $T$ with the critical theoretical value $T_{c}$. If $T \leq T_{c}$ the assumption that the empirical data $x_{1}, \ldots, x_{n}$ were drawn from the probability distribution $\rho(x)$ can be rejected with a confidence level of $100(1-\alpha) \%$. If $T \geq T_{c}$ there are no statistically significant reasons to reject the assumption. This is usually expressed in short saying that the sample follows the distribution $\rho(x)$ with $100(1-\alpha) \%$ confidence level.

\subsection{The Birge factor}

When $\chi_{\min }^{2} / \nu$ is outside the expected $1 \sigma$ confidence interval, $1 \pm \sqrt{2 / \nu}$, an artificial and global rescaling enlarging the errors is often recommended 21. From this point of view the long struggle of selecting a NN database for a high quality phenomenological potential [16, 17, 18, 19, 20, 22, 23, 24, 25] would be meaningless. However, as shown in Ref. 25 this is reasonable once the normality of residuals has been tested. We illustrate this rescaling in Fig. 1 for the NN analysis on the light of the normality TS test at the $95 \%$-confidence level. For the complete $N=8125$ database analyzed with OPE-DS (complete references to published data are provided in Ref. [23]), a lack of normality is evident both before $\left(\chi^{2} / \nu=1.4\right)$ and after rescaling. However, the $N=61733 \sigma$-mutually self-consistent selected database complies with normality before $\left(\chi^{2} / \nu=1.04\right)$ and after rescaling when analyzed with OPE-DS. This same database only complies with normality after rescaling when analyzed with $\chi$ TPE-DS (before rescaling $\chi^{2} / \nu=1.07$ ) 24]. This shows that rescaling is only justified provided normality of residuals has been achieved 25. 


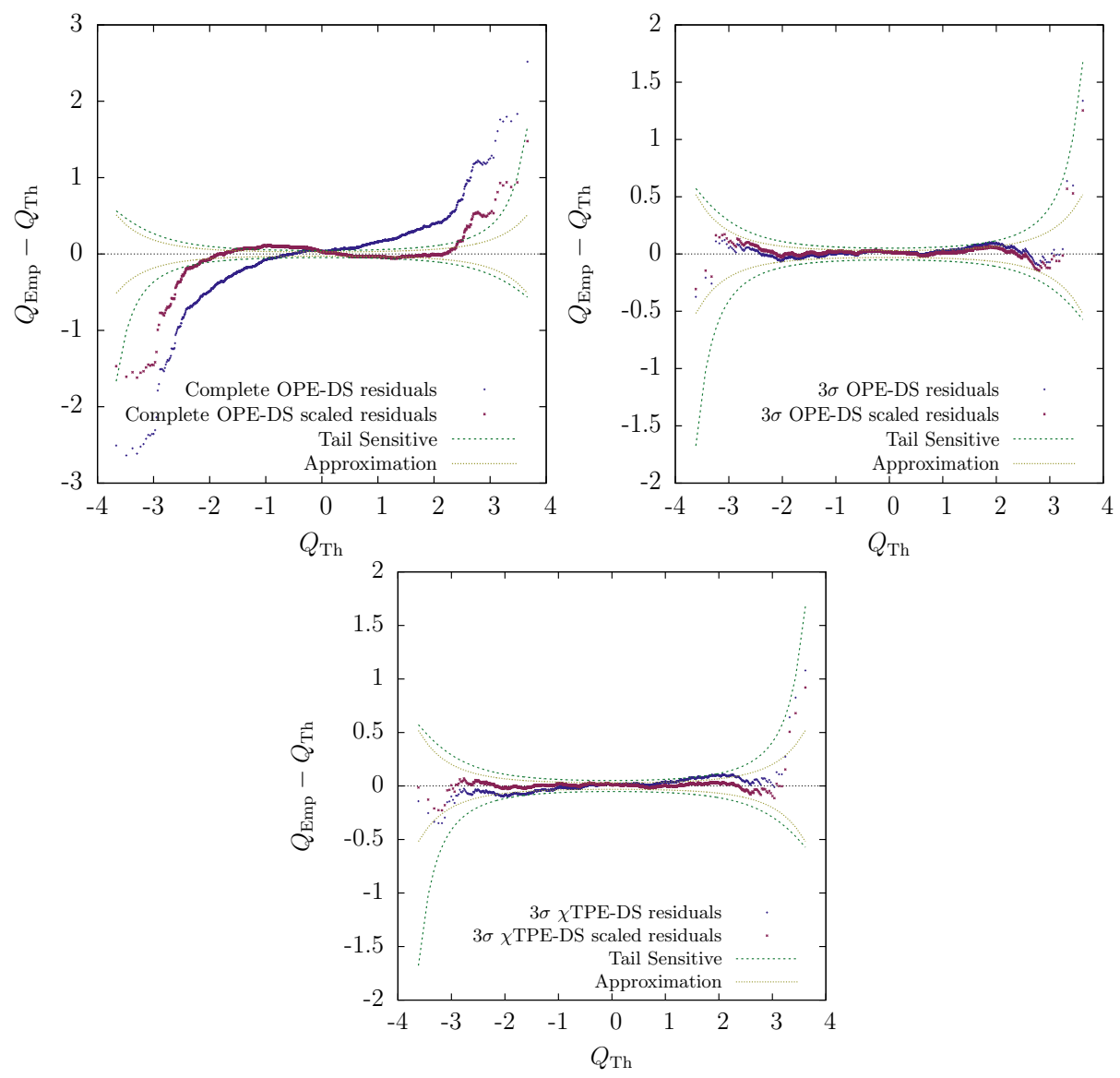

Figure 1. (Color online) Rotated QQ-plots with point-wise, Eq. 18, and TS 95\%-confidence bands compared with data residuals before and after Birge rescaling of errors. Top-left panel: Full database with $N=8125$ data residuals with OPE-DS 23. Top-right panel: Selected database with $N=6173$ date residuals with OPE-DS [23]. Lower panel: Selected database with $N=6173$ date residuals with $\chi$ TPE-DS [24].

\section{Effective interactions and their uncertainties}

\subsection{Motivation}

An important result from the statistical correlation analysis is that we may actually learn what is the most convenient scheme to search for the optimized interaction. Obviously, by diagonalizing the correlation matrix $\mathcal{C}_{i j}$ we may determine what are the linear combinations of fitting parameters which behave independently along the minimization path. However, this is inconvenient as does not precisely correspond to clear picture in the parameter space. In what follows we study this issue from the point of view of effective interactions as applied to the OPE-DS [23] and $\chi$ TPE-DS 24] interactions. We are using the same $3 \sigma$ self-consistent database of $N=6713$ data of Ref. 23] which as described above passes satisfactorily the TS normality test 25]. 


\subsection{Moshinsky-Skyrme parameters}

Effective interactions in Nuclear Physics were proposed by Moshinsky [44 and Skyrme [45]. As compared to ab initio calculations, the nuclear many body wave function has a much simpler structure since short range correlations play a marginal role allowing for a fruitful implementation of mean field Hartree-Fock calculations [46, 47. 48. At the two body level the effective interaction of Moshinsky 44 and Skyrme 45] can be written as a pseudo-potential in the form

$$
\begin{aligned}
& V_{\Lambda}\left(\mathbf{p}^{\prime}, \mathbf{p}\right)=\int d^{3} x e^{-i \mathbf{x} \cdot\left(\mathbf{p}^{\prime}-\mathbf{p}\right)} \hat{V}(\mathbf{x}) \\
& =t_{0}\left(1+x_{0} P_{\sigma}\right)+\frac{t_{1}}{2}\left(1+x_{1} P_{\sigma}\right)\left(\mathbf{p}^{\prime 2}+\mathbf{p}^{2}\right) \\
& +t_{2}\left(1+x_{2} P_{\sigma}\right) \mathbf{p}^{\prime} \cdot \mathbf{p}+2 i W_{0} \mathbf{S} \cdot\left(\mathbf{p}^{\prime} \wedge \mathbf{p}\right) \\
& +\frac{t_{T}}{2}\left[\sigma_{1} \cdot \mathbf{p} \sigma_{2} \cdot \mathbf{p}+\sigma_{1} \cdot \mathbf{p}^{\prime} \sigma_{2} \cdot \mathbf{p}^{\prime}-\frac{1}{3} \sigma_{1} \cdot \sigma_{2}\left(\mathbf{p}^{\prime 2}+\mathbf{p}^{2}\right)\right] \\
& +\frac{t_{U}}{2}\left[\sigma_{1} \cdot \mathbf{p} \sigma_{2} \cdot \mathbf{p}^{\prime}+\sigma_{1} \cdot \mathbf{p}^{\prime} \sigma_{2} \cdot \mathbf{p}-\frac{2}{3} \sigma_{1} \cdot \sigma_{2} \mathbf{p}^{\prime} \cdot \mathbf{p}\right]+\mathcal{O}\left(p^{4}\right)
\end{aligned}
$$

where $P_{\sigma}=\left(1+\sigma_{1} \cdot \sigma_{2}\right) / 2$ is the spin exchange operator with $P_{\sigma}=-1$ for spin singlet $(S=0)$, and $P_{\sigma}=1$ for spin triplet $(S=1)$ states. The cut-off $\Lambda$ specifies the maximal CM momentum scale, and $\Delta r=\hbar / \Lambda$ the de Broglie resolution. The scale dependence of the parameters was determined in Ref. 32 just from NN threshold properties such as scattering lengths, effective ranges and volumes without explicitly taking into account the finite range of the NN interaction, a procedure which is well justified for $\Lambda \lesssim 200 \mathrm{MeV}$ when the Similarity Renormalization Group is invoked [34]. One can likewise take $\Lambda$ as the maximal fitting CM momentum [35]. Here we will take the fixed value $\Lambda=400 \mathrm{MeV}$ and exemplify our results for both OPE-DS 23 and $\chi$ TPE-DS [24]. The expansion contains just those 9 parameters which can be determined from two-body data alone 32. Using the formulas from 35 expressing the Skyrme parameters as volume integrals we get the numerical results of Table 1 . We separate the contributions stemming from the inner region $r<r_{c}$ containing just delta-shell interactions and the outer region $r>r_{c}$ containing the pion exchange

potential tail. It is noteworthy the numerical agreement between the full integrals of both potentials. In Fig. 2 we show the correlation matrices corresponding to the inner parts. In both cases correlations are small.

As mentioned and demonstrated in 35] the numerical values of the parameters depend on the resolution scale. Therefore, a direct numerical comparison of our values with mean field approaches should not be taken as a measure of agreement; actually we expect that a suitable $\Lambda \sim 100-200 \mathrm{MeV}$ would produce closer numbers 32 .

\subsection{Counterterms and their uncertainties}

The potential in momentum space can be written in the partial wave basis as

$$
v_{l^{\prime}, l}^{J S}\left(p^{\prime}, p\right)=(4 \pi)^{2} \int_{0}^{\infty} d r r^{2} j_{l^{\prime}}\left(p^{\prime} r\right) j_{l}(p r) V_{l^{\prime} l}^{J S}(r)
$$

Using the Bessel function expansion for small argument $j_{l}(x)=x^{l} /(2 l+1)$ !! [1 $x^{2} / 2(2 l+3)+\ldots$. we get a low momentum expansion of the potential matrix elements. We keep up to total order $\mathcal{O}\left(p^{4}, p^{\prime 4}, p^{2} p^{2}\right)$ corresponding to $S$-, $P$ - and $D$-waves as 
Table 1. Moshinsky-Skyrme parameters for the renormalization scale $\Lambda=$ $400 \mathrm{MeV}$. We separate the contribution from the delta-shells short range parameters (corresponding to $r<r_{c}$ ) and the potential tail (corresponding to $\left.r>r_{c}\right)$ both for OPE-DS $\left(r_{c}=3 \mathrm{fm}\right)$ 23] and $\chi$ TPE-DS $\left(r_{c}=1.8 \mathrm{fm}\right)$ 24]. Units are: $t_{0}$ in $\mathrm{MeVfm}^{3}, t_{1}, t_{2}, W_{0}, t_{U}, t_{T}$ in $\mathrm{MeVfm}^{5}$, and $x_{0}, x_{1}, x_{2}$ are dimensionless.

\begin{tabular}{ccccccc}
\hline & \multicolumn{3}{c}{ OPE-DS $\left(r_{c}=3 \mathrm{fm}\right)$} & \multicolumn{3}{c}{$\chi \mathrm{TPE}-\mathrm{DS}\left(r_{c}=1.8 \mathrm{fm}\right)$} \\
& $r<r_{c}$ & $r>r_{c}$ & Full & $r<r_{c}$ & $r>r_{c}$ & Full \\
\hline$t_{0}$ & $-490.6(64)$ & -136.2 & $-626.8(64)$ & $-170.9(70)$ & $-358.7(32)$ & $-529.6(53)$ \\
$x_{0}$ & $-0.49(2)$ & 0.032 & $-0.38(2)$ & $-1.55(7)$ & $-0.0934(8)$ & $-0.56(1)$ \\
$t_{1}$ & $357.7(30)$ & 590.4 & $948.1(30)$ & $114.7(29)$ & $798.8(21)$ & $913.6(22)$ \\
$x_{1}$ & $-0.218(9)$ & 0.055 & $-0.048(3)$ & $-0.53(2)$ & $-0.00855(2)$ & $-0.074(3)$ \\
$t_{2}$ & $407.5(56)$ & 2055.1 & $2462.6(56)$ & $230.6(31)$ & $2259.4(46)$ & $2490.0(39)$ \\
$x_{2}$ & $-1.118(4)$ & -0.8190 & $-0.8686(6)$ & $-0.71(1)$ & $-0.892(1)$ & $-0.8750(8)$ \\
$W_{0}$ & $107.7(4)$ & 0 & $107.7(4)$ & $96.1(4)$ & 4.7 & $100.8(3)$ \\
$t_{U}$ & $392.9(12)$ & 885.7 & $1278.6(12)$ & $127.8(6)$ & $1132.5(7)$ & $1260.3(5)$ \\
$t_{T}$ & $-1204.4(87)$ & -3016.5 & $-4220.9(87)$ & $-457.7(31)$ & $-3835.1(21)$ & $-4292.8(23)$ \\
\hline
\end{tabular}

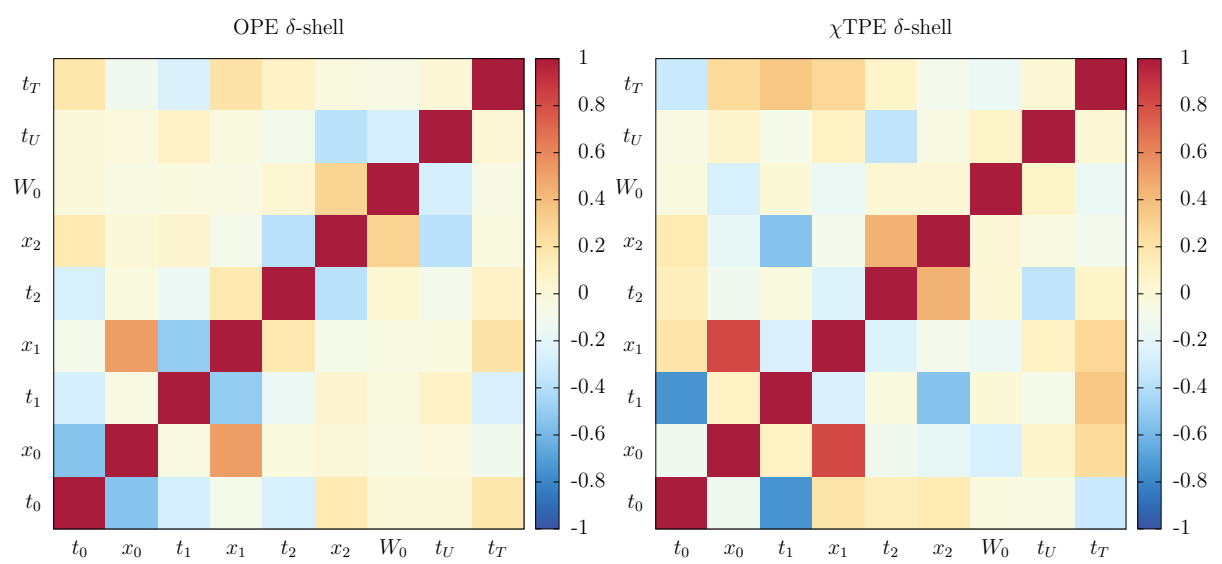

Figure 2. (Color online) Correlation matrix $\mathcal{C}_{i j}$, Eq. (4), for the 9 Skyrme parameters, see Eq. (23). We show the OPE-DS (left panel) and the $\chi$ TPE-DS (right panel) potentials. We grade from $100 \%$ correlation, $\mathcal{C}_{i j}=1$ (dark red), $0 \%$ correlation, $\mathcal{C}_{i j}=0$ (light yellow) and $100 \%$ anti-correlation, $\mathcal{C}_{i j}=-1$ (dark blue).

well as S-D and P-F mixing parameters,

$$
\begin{aligned}
& v_{00}^{J S}\left(p^{\prime}, p\right)=\widetilde{C}_{00}^{J S}+C_{00}^{J S}\left(p^{2}+p^{2}\right)+D_{00}^{1}{ }^{J S}\left(p^{4}+p^{\prime 4}\right)+D_{00}^{2}{ }^{J S} p^{2} p^{2}+\cdots \\
& v_{11}^{J S}\left(p^{\prime}, p\right)=p p^{\prime} C_{11}^{J S}+p p^{\prime}\left(p^{2}+p^{\prime 2}\right) D_{11}^{J S}+\cdots \\
& v_{22}^{J S}\left(p^{\prime}, p\right)=p^{2} p^{\prime 2} D_{22}^{J S}+\cdots \\
& v_{20}^{J S}\left(p^{\prime}, p\right)=p^{\prime 2} C_{20}^{J S}+p^{\prime 2} p^{2} D_{20}^{1}{ }^{J S}+p^{\prime 4} D_{20}^{2}{ }^{J S}+\ldots
\end{aligned}
$$




$$
v_{31}^{J S}\left(p^{\prime}, p\right)=p^{\prime 3} p D_{31}^{J S}+\cdots
$$

We use the spectroscopic notation and normalization of Ref. 8 . We will call the coefficients in the expansion counterterms \&. Our numerical results are shown in table 2 for OPE-DS 23 and $\chi$ TPE-DS 24]. In both cases it is interesting to separate the short distance contribution from the explicit potential tail containing the pion contributions. As we see, while the separate contributions greatly differ from one region to another, there is a high degree of universality for the full integral, which accounts for the integrated strength of the interaction. The correlation matrices are presented in Fig. 3 for the inner $r<r_{c}$ contributions. The short distance contribution to the counterterms in the partial wave basis are largely independent, although the $\chi \mathrm{TPE}$ enhances correlations as compared to OPE, see Fig. 3. This correlation information is actually very useful to find a flexible minimization path in both OPE-DS 23. and $\chi$ TPE-DS 24] potentials and complies to the same correlation pattern obtained in our previous work 25. In any case it is easier to take the short distance parameters as primary fitting quantities and the counterterms as derived ones.

Note that a direct numerical comparison with momentum space treatments [8, 49] is tricky since the particular choice of splitting the pionic and short distance interaction is done differently. This can be seen in the numerical values given in 8 and 49 where the same convention and chiral potential is used but different fitting ranges are considered. In addition we find that there are systematic differences within our quoted statistical uncertainties, table 2 (no statistical errors are quoted in [8] and [49]). In all we show 24-low energy constants in the np case, but note that they stem from $42 \mathrm{np}$ parameters in the OPE case and 27 in the $\chi \mathrm{TPE}$ case, so we could have taken these low energy constants themselves as fitting parameters.
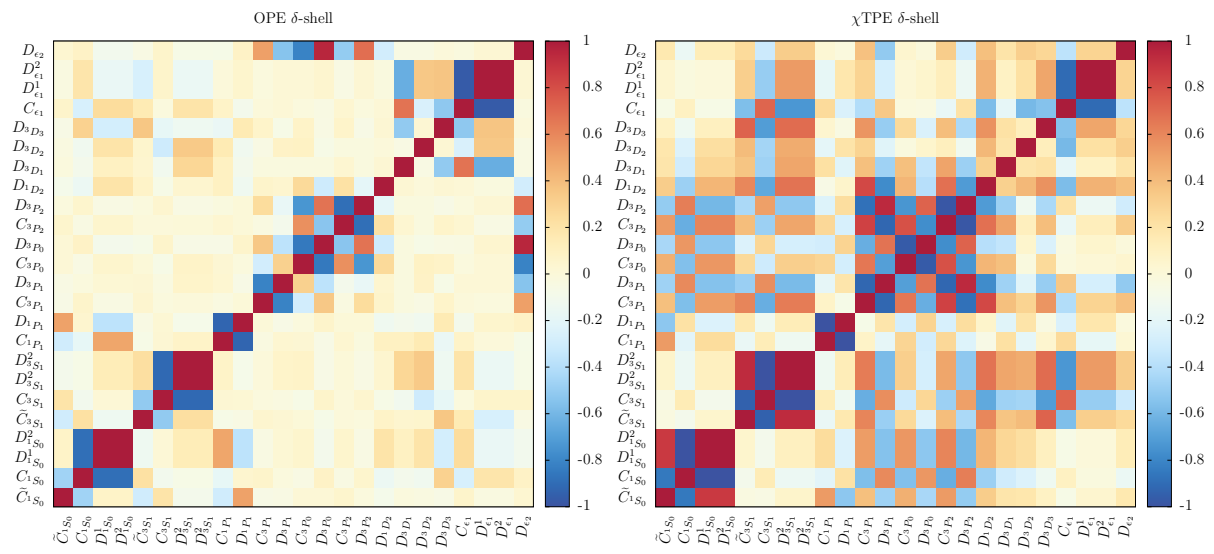

Figure 3. (Color online) Same as Fig 2 but for the short distance contribution to the 24 partial wave counterterms including up to $\mathcal{O}\left(p^{4}\right)$

$\S$ Properly speaking the name is justified when the potential $v\left(p^{\prime}, p\right)$ is used to solve the problem in a restricted Hilbert space $p, p^{\prime} \leq \Lambda$ which means in particular fitting scattering data up to CM momentum $p \leq \Lambda$ [34]. Only under these conditions is a truly universal behaviour of the counterterms guaranteed for $\Lambda \lesssim 1 / a$. 
Table 2. Potential integrals in different partial waves. We separate the contribution from the delta-shells short range parameters (corresponding to $r<r_{c}$ ) and the potential tail (corresponding to $r>r_{c}$ ) both for OPE-DS $\left(r_{c}=3 \mathrm{fm}\right)$ [23] and $\chi$ TPE-DS $\left(r_{c}=1.8 \mathrm{fm}\right)$ 24]. Units are: $\widetilde{C}$ 's are in $10^{4} \mathrm{GeV}^{-2}$, $C$ 's are in $10^{4} \mathrm{GeV}^{-4}$ and $D$ 's are in $10^{4} \mathrm{GeV}^{-6}$.

\begin{tabular}{|c|c|c|c|c|c|c|}
\hline & \multicolumn{3}{|c|}{$\mathrm{OPE}-\mathrm{DS}\left(r_{c}=3 \mathrm{fm}\right)$} & \multicolumn{3}{|c|}{$\chi \mathrm{TPE}-\mathrm{DS}\left(r_{c}=1.8 \mathrm{fm}\right)$} \\
\hline & $r<r_{c}$ & $r>r_{c}$ & Full & $r<r_{c}$ & $r>r_{c}$ & Full \\
\hline$\widetilde{C}_{{ } S_{0}}$ & $0.120(1)$ & -0.021 & $-0.141(1)$ & $-0.071(2)$ & $-0.0641(5)$ & $-0.135(2)$ \\
\hline$C_{{ }}$ & $1.83(2)$ & 2.34 & $4.17(2)$ & $.74(2)$ & $3.384(9)$ & $4.12(2)$ \\
\hline$D^{1} S_{0}$ & $-39.2(11)$ & -409.6 & $-448.8(11)$ & 5.1(3) & $-437.6(4)$ & $-443.7(5)$ \\
\hline$D_{1}^{2} S_{0}$ & - & -122.8 & $-134.6(3)$ & $.83(9)$ & $-131.3(1)$ & $-133.1(1)$ \\
\hline$\widetilde{C}_{{ }} S_{1}$ & $-0.041(2)$ & -0.023 & -0.06 & 2) & $-0.0532(5)$ & $-0.038(1)$ \\
\hline$C_{{ } S_{1}}$ & (2) & & (1, & 4 & & \\
\hline$D^{1} S_{1}$ & $-24.3(3)$ & -486.4 & $-510.7(3)$ & $-3.8(2)$ & $-500.9(4)$ & $-504.7(4)$ \\
\hline$D_{3 S_{1}}^{2}$ & -7 & -14 & -153. & 6) & -15 & -151 \\
\hline$C_{{ } P_{1}}$ & 1 . & 5 & $6.44(2)$ & r & 5 & 1) \\
\hline$D^{{ }} P_{1}$ & $-11.2(2)$ & -583.7 & $-594.9(2)$ & $-2.04(2)$ & $-590.1(2)$ & $-592.1(2)$ \\
\hline$C_{3} P_{1}$ & $1.151(3)$ & 2.587 & $.738(2)$ & & 2.9 & 3.6 \\
\hline$D{ } P_{1}$ & $-9.44(5)$ & -243.85 & $-253.29(5)$ & $-2.55(5)$ & $-247.3(1)$ & $-249.8(2)$ \\
\hline$C{ }^{3} P_{0}$ & $-1.298(8)$ & -3.613 & $-4.911(8)$ & 0.0 & -4 & $-4.882(5)$ \\
\hline$D^{3} P_{0}$ & $23.1(2)$ & 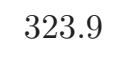 & 皮 &.$(0)$ & $342.5(2)$ & $343.6(2)$ \\
\hline$C_{3} P_{2}$ & $-0.552(2)$ & & -0.445 & & & $-0.434(3)$ \\
\hline$D{ } P_{2}$ & $6.14(7)$ & -16.76 & $-10.62(7)$ & & & $-9.7(2)$ \\
\hline$D^{{ }^{1}} D_{2}$ & $-5.38(3)$ & -65.54 & $-70.92(3)$ & $-0.65(3)$ & $-70.01(6)$ & $-70.66(6)$ \\
\hline$D^{{ }^{3} D_{2}}$ & $-17.0(2)$ & -350.8 & $-367.8(2)$ & $-1.87(3)$ & $-362.52(6)$ & $-364.39(7)$ \\
\hline$D^{{ }^{3} D_{1}}$ & $10.7(2)$ & 195.1 & $205.8(2)$ & $1.51(4)$ & $202.74(7)$ & $204.25(7)$ \\
\hline$D^{{ }^{3} D_{3}}$ & $0.41(1)$ & 0.14 & $0.55(1)$ & $0.54(1)$ & $0.33(6)$ & $0.87(6)$ \\
\hline$C_{\epsilon_{1}}$ & $-2.38(2)$ & -5.98 & $-8.36(2)$ & $-0.906(6)$ & $-7.594(4)$ & $-8.500(4)$ \\
\hline$D_{\epsilon_{1}}^{1}$ & $47.7(6)$ & 964.9 & $1012.6(6)$ & $6.73(7)$ & $998.8(1)$ & $1005.5(1)$ \\
\hline & $20.4(3)$ & 413.6 & $434.0(3)$ & $2.88(3)$ & $428.06(5)$ & $430.94(4)$ \\
\hline$D_{\epsilon_{2}}$ & $4.72(4)$ & 79.46 & $84.18(4)$ & $0.709(6)$ & $82.58(1)$ & $83.29(1)$ \\
\hline
\end{tabular}

\subsection{Discussion}

The universality and lack of correlation among fitting parameters are good features of a least squares minimization procedure. Our results above address the issue and a clear picture emerges. From an EFT point of view [3, 5, 7, 8, 9, the partial wave parameters are nothing but the needed counterterms at a given renormalization scale $\Lambda$, as they encode the integrated out short range information of the interaction. We can also see 
that the correlation matrix among the different counterterms indicates to what extent are they intertwined. This is important since in EFT counterterms are assumed to be independent variables. Discovering correlations among them means that some extra symmetry or condition has been overlooked. It is remarkable that at $\Lambda \sim 200 \mathrm{MeV}$ a long distance symmetry such as the spin-isospin SU(4)-Wigner invariance emerges from the NN data and simultaneously is expected from a large $N_{c}$ approach [11, 13, 14]. This pattern does not show up in Fig. 3 corresponding to $\Lambda=400 \mathrm{MeV}$ but without restricting the full Hilbert space for off-shell momenta $p, p^{\prime} \leq \Lambda$. This suggests a correlation analysis closer in spirit to the BD-SRG approach 31, 34.

\section{Conclusions}

There is currently an unbalanced accuracy between theory and experiment in Nuclear Physics. This is due not only to the complexity of the nuclear many body problem but also because the fundamental nucleon-nucleon interaction is not known precisely in the mid-range region which is the relevant one for nuclear binding. On top of this the precise level of inaccuracy of the nuclear force is rarely estimated. This is a major handicap for the assessment of theoretical uncertainties when going from the two body problem to the many body situation. At present, the most accurate source of information of the two body interaction is the large collection of about $8000 \mathrm{np}$ and pp scattering data, which by themselves are subjected to experimental uncertainties. The disentanglement of systematic and statistical errors, while challenging in itself, can be simplified by selecting scattering data which are not only mutually consistent but also true normal fluctuations with respect to the most likely phenomenological interaction. This desirable scenario, while is not at all guaranteed, does actually happen in our analysis and furnishes the necessary requirements for a sound statistical error propagation. We have discussed with some detail the statistical considerations to test the normality of residuals with a stringent and recently proposed tail sensitive scheme. We also provide a straightforward recipe to carry out such a useful test for any least squares fit. As a particular but insightful application, we have also profited from the applicability of statistical error and correlation analysis to quantify the uncertainty of effective interactions defined as volume integrals of the potential. As we have noted, effective interactions depend on the resolution scale determined by the shortest de Broglie wavelength involved. We have focused and restricted on a relatively small distance scale determined by the pion production threshold, where the repulsive short distance features become relevant. As preliminary calculations show the scale dependence could actually be tuned to the relevant de Broglie wavelength dominating the NN interaction in finite nuclei. In any case a certain degree of universality emerges from our analysis, and reinforces the view that effective interaction parameters have desirable features of fitting parameters, namely small statistical correlations and scheme independence.

This work is supported by Spanish DGI (grant FIS2011-24149) and Junta de Andalucía (grant FQM225). R.N.P. is supported by a Mexican CONACYT grant.

\section{Appendix A. Tables on Tail Sensitive Test Statistic}

The critical values in table A1 were obtained by taking $M=500000$ random samples with size $N$ of uniformly distributed data. The rather high number of samples was 
Table A1. Critical values of $T_{\mathrm{c}}$ for the Tail Sensitive normality test as a function of the sample size $N$ at different levels of significance $\alpha$. The critical values were obtained using MonteCarlo simulations taking 500000 samples for each value of $N$ between 1 and 50 .

\begin{tabular}{|c|c|c|c|c|c|}
\hline$N \backslash \alpha$ & 0.01 & 0.02 & 0.05 & 0.10 & 0.20 \\
\hline 1 & 0.01001773 & 0.02009725 & 0.05004725 & 0.10012406 & 0.19996581 \\
\hline 2 & 0.00513134 & 0.01036312 & 0.02648217 & 0.05448341 & 0.11389617 \\
\hline 3 & 0.00351999 & 0.00718572 & 0.01862308 & 0.03892356 & 0.08284280 \\
\hline 4 & 0.00274579 & 0.00559971 & 0.01464807 & 0.03088211 & 0.06658627 \\
\hline 5 & 0.00225585 & 0.00466067 & 0.01228834 & 0.02606941 & 0.05682341 \\
\hline 6 & 0.00194960 & 0.00403137 & 0.01064535 & 0.02273282 & 0.04997837 \\
\hline 7 & 0.00171087 & 0.00355567 & 0.00950508 & 0.02041778 & 0.04507288 \\
\hline 8 & 0.00153800 & 0.00320521 & 0.00862923 & 0.01856308 & 0.04123497 \\
\hline 9 & 0.00142940 & 0.00296436 & 0.00796641 & 0.01716920 & 0.03823687 \\
\hline 10 & 0.00130562 & 0.00272923 & 0.00739200 & 0.01599717 & 0.03579932 \\
\hline 11 & 0.00121215 & 0.00255493 & 0.00693178 & 0.01504779 & 0.03371454 \\
\hline 12 & 0.00115210 & 0.00241817 & 0.00654682 & 0.01423879 & 0.03203655 \\
\hline 13 & 0.00108994 & 0.00229341 & 0.00620929 & 0.01353055 & 0.03054902 \\
\hline 14 & 0.00103473 & 0.00217373 & 0.00592355 & 0.01295297 & 0.02922865 \\
\hline 15 & 0.00098603 & 0.00208026 & 0.00568029 & 0.01243297 & 0.02814438 \\
\hline 16 & 0.00094095 & 0.00199030 & 0.00545322 & 0.01193342 & 0.02711705 \\
\hline 17 & 0.00091111 & 0.00192155 & 0.00524212 & 0.01153270 & 0.02622548 \\
\hline 18 & 0.00087656 & 0.00185463 & 0.00508330 & 0.01116882 & 0.02539659 \\
\hline 19 & 0.00084824 & 0.00178377 & 0.00490132 & 0.01079536 & 0.02462677 \\
\hline 20 & 0.00081993 & 0.00173589 & 0.00475800 & 0.01049558 & 0.02399643 \\
\hline 21 & 0.00079345 & 0.00168404 & 0.00464703 & 0.01023746 & 0.02338279 \\
\hline 22 & 0.00077672 & 0.00164040 & 0.00451678 & 0.00995987 & 0.02278223 \\
\hline 23 & 0.00075279 & 0.00159405 & 0.00441474 & 0.00974473 & 0.02233426 \\
\hline 24 & 0.00073934 & 0.00156243 & 0.00430510 & 0.00953482 & 0.02183490 \\
\hline 25 & 0.00071677 & 0.00151937 & 0.00421339 & 0.00930246 & 0.02137357 \\
\hline 26 & 0.00069494 & 0.00148552 & 0.00412071 & 0.00912411 & 0.02092654 \\
\hline 27 & 0.00068503 & 0.00145519 & 0.00401387 & 0.00891337 & 0.02055165 \\
\hline 28 & 0.00066741 & 0.00141934 & 0.00394817 & 0.00875921 & 0.02018670 \\
\hline 29 & 0.00065925 & 0.00139673 & 0.00387957 & 0.00861749 & 0.01982410 \\
\hline 30 & 0.00064347 & 0.00137429 & 0.00380204 & 0.00845258 & 0.01949674 \\
\hline 31 & 0.00063372 & 0.00134370 & 0.00374100 & 0.00830829 & 0.01920333 \\
\hline 32 & 0.00062434 & 0.00133204 & 0.00368650 & 0.00819084 & 0.01892001 \\
\hline 33 & 0.00061108 & 0.00130865 & 0.00362774 & 0.00805833 & 0.01865113 \\
\hline 34 & 0.00060333 & 0.00128911 & 0.00357679 & 0.00796122 & 0.01842678 \\
\hline 35 & 0.00059576 & 0.00126498 & 0.00352836 & 0.00784902 & 0.01816494 \\
\hline 36 & 0.00058123 & 0.00124395 & 0.00347131 & 0.00772213 & 0.01790285 \\
\hline 37 & 0.00057221 & 0.00122973 & 0.00342757 & 0.00764118 & 0.01770791 \\
\hline 38 & 0.00056670 & 0.00120748 & 0.00336587 & 0.00752593 & 0.01744823 \\
\hline 39 & 0.00056443 & 0.00119716 & 0.00334196 & 0.00744526 & 0.01726910 \\
\hline 40 & 0.00055411 & 0.00118668 & 0.00330584 & 0.00735387 & 0.01704568 \\
\hline 41 & 0.00054087 & 0.00116426 & 0.00324657 & 0.00726325 & 0.01688035 \\
\hline 42 & 0.00053338 & 0.00114686 & 0.00321383 & 0.00717868 & 0.01668878 \\
\hline 43 & 0.00053060 & 0.00113421 & 0.00317425 & 0.00710159 & 0.01651373 \\
\hline 44 & 0.00052550 & 0.00113043 & 0.00315774 & 0.00702840 & 0.01634427 \\
\hline 45 & 0.00051907 & 0.00110859 & 0.00311188 & 0.00695394 & 0.01619440 \\
\hline 46 & 0.00051066 & 0.00109194 & 0.00307711 & 0.00689179 & 0.01605360 \\
\hline 47 & 0.00050927 & 0.00109077 & 0.00304886 & 0.00681100 & 0.01583361 \\
\hline 48 & 0.00050123 & 0.00107538 & 0.00301819 & 0.00675336 & 0.01569550 \\
\hline 49 & 0.00049858 & 0.00107373 & 0.00298081 & 0.00668439 & 0.01557817 \\
\hline 50 & 0.00049267 & 0.00105755 & 0.00296376 & 0.00663403 & 0.01544530 \\
\hline
\end{tabular}




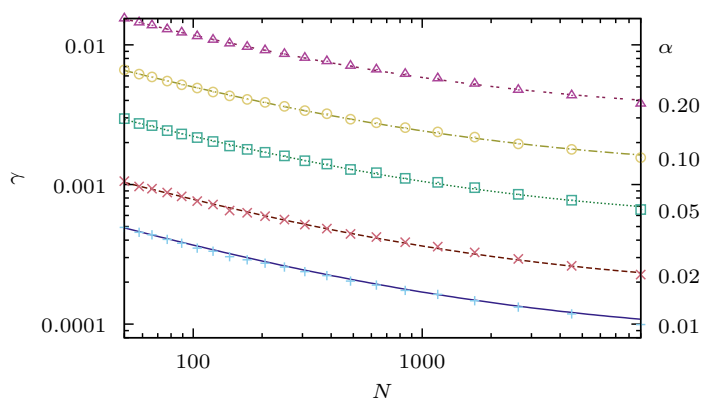

Figure A1. (Color online) Critical values for the Tail Sensitive normality test for large values of $\mathrm{N}$ at different levels of significance $\alpha$. The points where obtained via MonteCarlo simulations taking 500000 samples for each value of $N$. The curves correspond to the parameterization $T_{\mathrm{c}}=a / \sqrt{N}+b$; the fitted values for $a$ and $b$ at each level of significance are given in table $\mathrm{A2}$

Table A2. Large $N$ parameterization of the critical values for the Tail Sensitive normality test $T_{\mathrm{c}}=a / \sqrt{N}+b$ at different levels of significance. This parameterization is appropriate for $50<N \leq 9000$

\begin{tabular}{ccc}
$\alpha$ & $\mathrm{a}$ & $\mathrm{b}$ \\
\hline 0.01 & 0.0029065 & 0.0000728 \\
0.02 & 0.0061401 & 0.0001690 \\
0.05 & 0.0169933 & 0.0005161 \\
0.10 & 0.0377146 & 0.0012314 \\
0.20 & 0.0866187 & 0.0031098 \\
\hline
\end{tabular}

necessary to reduced the statistical fluctuations enough so that if $N_{1}<N_{2} \leq 50$ then $T_{1 c, \mathrm{TS}}>T_{2 c, \mathrm{TS}}$. One of the appeals of the Kolmogorov-Smirnov test is the simple $\sim 1 / \sqrt{N}$ parameterization of the critical values for large $N$. In Ref. 43 the authors proposed a $\sim \log (\log (N)) / \sqrt{N}$ limiting behavior, but the parameters values were not determined. We performed new Monte-Carlo simulations, maintaining the number of samples, to obtain the reliable critical values for several values of $N$ between 50 and 9000 and find a valid parameterization in this range of $N$. We tried the proposed parameterization by Aldor-Noiman et al. but found a better fit to our results, shown in Fig. A1, by using

$$
T_{c, \mathrm{TS}}=\frac{a}{\sqrt{N}}+b .
$$

The fitted parameters for different levels of significance are presented in table A2. It is important to mention that this parameterization is valid in the $50<N \leq 9000$ range and extrapolations to $N>9000$ may not be correct

[1] Ulf-G. Meißner. Nuclear forces and ab initio calculations of atomic nuclei. nucl-th/1401.5307, 2014.

[2] S. Aoki. Hadron interactions in lattice QCD. Prog.Part.Nucl.Phys., 66:687-726, 2011.

[3] Steven Weinberg. Nuclear forces from chiral Lagrangians. Phys.Lett., B251:288-292, 1990.

[4] C. Ordonez, L. Ray, and U. van Kolck. Nucleon-nucleon potential from an effective chiral Lagrangian. Phys.Rev.Lett., 72:1982-1985, 1994. 
[5] Norbert Kaiser, R. Brockmann, and W. Weise. Peripheral nucleon-nucleon phase shifts and chiral symmetry. Nucl.Phys., A625:758-788, 1997.

[6] M.C.M. Rentmeester, R.G.E. Timmermans, James Lewis Friar, and J.J. de Swart. Chiral two pion exchange and proton proton partial wave analysis. Phys.Rev.Lett., 82:4992-4995, 1999.

[7] D.R. Entem and R. Machleidt. Accurate charge dependent nucleon nucleon potential at fourth order of chiral perturbation theory. Phys.Rev., C68:041001, 2003.

[8] E. Epelbaum, W. Glockle, and Ulf-G. Meissner. The Two-nucleon system at next-to-next-tonext-to-leading order. Nucl.Phys., A747:362-424, 2005.

[9] R. Machleidt and D.R. Entem. Chiral effective field theory and nuclear forces. Phys.Rept., 503:1-75, 2011.

[10] H. Muther, C.A. Engelbrecht, and G.E. Brown. Nuclear Physics in Colorful Worlds. Nucl.Phys., A462:701-726, 1987.

[11] David B. Kaplan and Aneesh V. Manohar. The Nucleon-nucleon potential in the $1 / \mathrm{N}(\mathrm{c})$ expansion. Phys.Rev., C56:76-83, 1997.

[12] Manoj K. Banerjee, Thomas D. Cohen, and Boris A. Gelman. The Nucleon nucleon interaction and large N(c) QCD. Phys.Rev., C65:034011, 2002.

[13] A. Calle Cordon and E. Ruiz Arriola. Wigner symmetry, Large N(c) and Renormalized One Boson Exchange Potential. Phys.Rev., C78:054002, 2008.

[14] A. Calle Cordon and E. Ruiz Arriola. Serber symmetry, Large N(c) and Yukawa-like One Boson Exchange Potentials. Phys.Rev., C80:014002, 2009.

[15] R. Navarro Perez, J.E. Amaro, and E. Ruiz Arriola. Coarse graining Nuclear Interactions. Prog.Part.Nucl.Phys., 67:359-364, 2012.

[16] V.G.J. Stoks, R.A.M. Kompl, M.C.M. Rentmeester, and J.J. de Swart. Partial wave analaysis of all nucleon-nucleon scattering data below 350-MeV. Phys.Rev., C48:792-815, 1993.

[17] V.G.J. Stoks, R.A.M. Klomp, C.P.F. Terheggen, and J.J. de Swart. Construction of high quality N N potential models. Phys.Rev., C49:2950-2962, 1994.

[18] Robert B. Wiringa, V.G.J. Stoks, and R. Schiavilla. An Accurate nucleon-nucleon potential with charge independence breaking. Phys.Rev., C51:38-51, 1995.

[19] R. Machleidt. The High precision, charge dependent Bonn nucleon-nucleon potential (CDBonn). Phys.Rev., C63:024001, 2001.

[20] Franz Gross and Alfred Stadler. Covariant spectator theory of np scattering: Phase shifts obtained from precision fits to data below 350-MeV. Phys.Rev., C78:014005, 2008.

[21] J. Dobaczewski, W. Nazarewicz, and P. G. Reinhard. Error Estimates of Theoretical Models: a Guide. Journal of Physics G: Nuclear and Particle Physics, 41(7):074001, 2014.

[22] R. Navarro Perez, J.E. Amaro, and E. Ruiz Arriola. Partial Wave Analysis of Nucleon-Nucleon Scattering below pion production threshold. Phys.Rev., C88:024002, 2013.

[23] R. Navarro Perez, J.E. Amaro, and E. Ruiz Arriola. Coarse grained Potential analysis of neutron-proton and proton-proton scattering below pion production threshold. Phys.Rev., C88:064002, 2013.

[24] R. Navarro Perez, J.E. Amaro, and E. Ruiz Arriola. Coarse grained NN potential with Chiral Two Pion Exchange. Phys.Rev., C89:024004, 2014.

[25] R. Navarro Perez, J.E. Amaro, and E. Ruiz Arriola. Statistical Error analysis of Nucleon-Nucleon phenomenological potentials. nucl-th/1404.0314, 2014.

[26] Michael J Evans and Jeffrey S Rosenthal. Probability and statistics: The science of uncertainty. Macmillan, 2004.

[27] Fred James and Matthias Winkler. Minuit users guide. CERN, Geneva, 2004.

[28] M. Kortelainen, T. Lesinski, J. More, W. Nazarewicz, J. Sarich, et al. Nuclear Energy Density Optimization. Phys.Rev., C82:024313, 2010.

[29] S.K. Bogner, T.T.S. Kuo, A. Schwenk, D.R. Entem, and R. Machleidt. Towards a model independent low momentum nucleon nucleon interaction. Phys.Lett., B576:265-272, 2003.

[30] S.K. Bogner, R.J. Furnstahl, and A. Schwenk. From low-momentum interactions to nuclear structure. Prog.Part.Nucl.Phys., 65:94-147, 2010.

[31] E. Anderson, S.K. Bogner, R.J. Furnstahl, E.D. Jurgenson, R.J. Perry, et al. Block Diagonalization using SRG Flow Equations. Phys.Rev., C77:037001, 2008.

[32] E. Ruiz Arriola. Low scale saturation of Effective NN Interactions and their Symmetries. nuclth/1009.4161, 2010.

[33] E. Ruiz Arriola. Van der Waals forces and Photon-less Effective Field Theories. Few Body Syst., 50:399-402, 2011.

[34] E. Ruiz Arriola, S. Szpigel, and V.S. Timoteo. Implicit vs Explicit Renormalization and Effective Interactions. Phys.Lett., B728:596-601, 2014.

[35] R. Navarro Perez, J.E. Amaro, and E. Ruiz Arriola. Effective Interactions in the Delta-Shells 
Potential. Few Body Syst., 54:1487-1490, 2013.

[36] R. Navarro Perez, J.E. Amaro, and E. Ruiz Arriola. Error estimates on Nuclear Binding Energies from Nucleon-Nucleon uncertainties. nucl-th/1202.6624, 2012.

[37] R. Navarro Perez, J.E. Amaro, and E. Ruiz Arriola. Nuclear Binding Energies and NN uncertainties. PoS, QNP2012:145, 2012.

[38] J.E. Amaro, R. Navarro Perez, and E. Ruiz Arriola. Error Analysis of Nuclear Matrix Elements. Few-Body Systems, 2013.

[39] J. Toivanen, J. Dobaczewski, M. Kortelainen, and K. Mizuyama. Error analysis of nuclear mass fits. Phys.Rev., C78:034306, 2008.

[40] J Dudek, B Szpak, B Fornal, and A Dromard. Predictive power and theoretical uncertainties of mathematical modelling for nuclear physics. Physica Scripta, 2013(T154):014002, 2013.

[41] R. Navarro Perez, J. E. Amaro and E. Ruiz Arriola, Bootstrapping the statistical uncertainties of NN scattering data arXiv:1407.3937 [nucl-th].

[42] R. Navarro Perez, E. Garrido, J. E. Amaro and E. Ruiz Arriola, Triton binding energy with realistic precision arXiv:1407.7784 [nucl-th].

[43] Sivan Aldor-Noiman, Lawrence D. Brown, Andreas Buja, Wolfgang Rolke, and Robert A. Stine. The power to see: A new graphical test of normality. The American Statistician, 67(4):249260, 2013.

[44] M. Moshinsky. Short range forces and nuclear shell theory. Nuclear Physics, 8:19 - 40, 1958.

[45] T. Skyrme. The effective nuclear potential. Nucl.Phys., 9:615-634, 1959.

[46] D. Vautherin and D.M. Brink. Hartree-Fock calculations with Skyrme's interaction. 1. Spherical nuclei. Phys.Rev., C5:626-647, 1972.

[47] E. Chabanat, J. Meyer, P. Bonche, R. Schaeffer, and P. Haensel. A Skyrme parametrization from subnuclear to neutron star densities. Nucl.Phys., A627:710-746, 1997.

[48] Michael Bender, Paul-Henri Heenen, and Paul-Gerhard Reinhard. Self-consistent mean-field models for nuclear structure. Rev.Mod.Phys., 75:121-180, 2003.

[49] A. Ekström, G. Baardsen, C. Forssén, G. Hagen, M. Hjorth-Jensen, et al. An optimized chiral nucleon-nucleon interaction at next-to-next-to-leading order. Phys.Rev.Lett., 110:192502, 2013. 